EPJ Web of Conferences 43, 01006 (2013)

DOI: $10.1051 /$ epjconf/20134301006

(C) Owned by the authors, published by EDP Sciences, 2013

\title{
Thermohaline instability and rotation-induced mixing in low and intermediate mass stars: Consequences on global asteroseismic quantities
}

\author{
N. Lagarde ${ }^{1, a}$, P. Eggenberger ${ }^{1}$, C. Charbonnel ${ }^{1,2}$, T. Decressin $^{1}$, \\ S. Ekström ${ }^{1}$ and A. Palacios ${ }^{3}$ \\ ${ }^{1}$ Geneva Observatory, University of Geneva, Chemin des Maillettes 51, 1290 Versoix, \\ Switzerland \\ ${ }^{2}$ IRAP, UMR 5277 CNRS and Université de Toulouse, 14 Av. E. Belin, 31400 Toulouse, \\ France \\ ${ }^{3}$ LUPM, Université Montpellier II, CNRS, UMR 5299, Place E. Bataillon, 34095 Montpellier, \\ France
}

\begin{abstract}
Thermohaline mixing has been recently identified as the probable dominating process that governs the photospheric composition of low-mass bright red giant stars. Here, we present the predictions of stellar models computed with the code STAREVOL including this process together with rotational mixing. We compare our theoretical predictions with recent observations, and discuss the effects of both mechanisms on asteroseismic diagnostics.
\end{abstract}

\section{INTRODUCTION}

At all stages of their evolution, low- and intermediate-mass stars (LIMS) exhibit the signatures of complex physical processes that require challenging modelling beyond canonical (or standard) stellar theory (by canonical we refer to the modelling of non-rotating, non-magnetic stars, in which convection is the only mechanism that drives mixing in stellar interiors).

Rotation has been shown to change the internal dynamics of these stars, by means of the transport of both angular momentum and chemical species through the action of meridional circulation and shear turbulence. Rotation-induced mixing results in the variations of surface chemical properties of LIMS during the main sequence which can explain observations in these stars (e.g. [1, 2]).

In addition, [3] identified thermohaline mixing as the process that may govern the surface abundances of LIMS evolving on the upper end of the red giant branch (RGB). In these stars, this doublediffusive instability is induced by the mean molecular weight inversion created by the ${ }^{3} \mathrm{He}\left({ }^{3} \mathrm{He}, 2 \mathrm{p}\right)^{4} \mathrm{He}$ reaction in the radiative layers between the convective envelope and the hydrogen burning shell [4]. This mechanism has a crucial impact on the chemical evolution of light elements in our Galaxy ([5, 6]) and should also be taken into account in the other topical astrophysical domains that use stellar models as input physics.

Thanks to CoRoT ([7]) and Kepler ([8]) space missions, a large number of asteroseismic observations are obtained for different kinds of stars. Asteroseismology paves the way to better understand stellar interior and provides valuable and independent constraints on current stellar models

\footnotetext{
ae-mail: Nadege.Lagarde@unige.ch
}

This is an Open Access article distributed under the terms of the Creative Commons Attribution License 2.0, which permits unrestricted use, distribution, and reproduction in any medium, provided the original work is properly cited. 


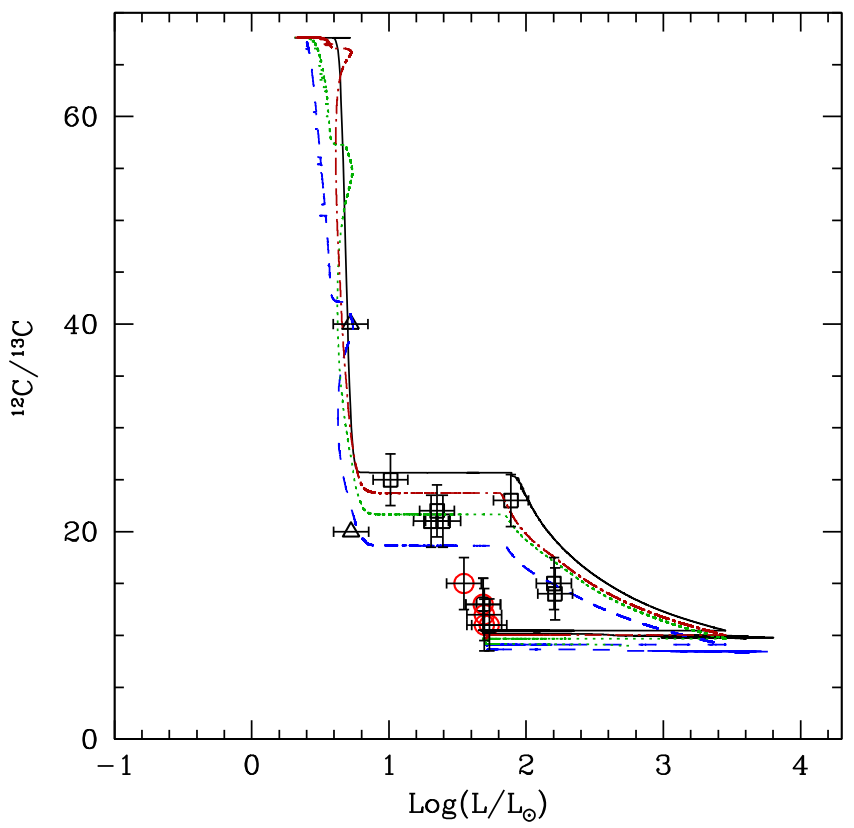

Figure 1. Evolution of the surface ${ }^{12} \mathrm{C} /{ }^{13} \mathrm{C}$ value as a function of stellar luminosity for the $1.25 \mathrm{M}_{\odot}$ models including thermohaline instability and rotation-induced mixing, for initial rotation velocities of 50, 80, and $110 \mathrm{~km} . \mathrm{s}^{-1}$ shown as dashed-dotted red, dotted green, and dashed blue lines respectively. The non-rotating case is also shown (black solid line). Observations along the evolutionary sequence of the open cluster M67 are from [10]. The triangle is for a subgiant star for which only a lower value could be obtained, while black squares and red circles correspond respectively to RGB and clump stars. Figure taken from [13].

as well as on the physics of different transport processes like rotation. We present here a grid of stellar models computed with the code STAREVOL by [9], including effects of rotation and thermohaline mixing on global asteroseismic quantities for a large range of masses $\left(0.85 \mathrm{M}_{\odot}\right.$ to $\left.6.0 \mathrm{M}_{\odot}\right)$ at different metallicities $(\mathrm{Z}=0.0001,0.002,0.004$, and 0.014$)$.

\section{EFFECTS OF THERMOHALINE INSTABILITY AND ROTATION-INDUCED MIXING ON SURFACE ABUNDANCES}

\subsection{Carbon isotopic ratio}

The behaviour of the carbon isotopic ratio is the best indicator of non-standard transport processes in evolved low-mass stars. This quantity has been determined in a large number of stars in Galactic open clusters. In Fig. 1 we compare the evolution of the theoretical surface carbon isotopic ratio for $1.25 \mathrm{M}_{\odot}$ models computed for three different initial velocities and including thermohaline mixing, with observations in M67 stars by [10] (turnoff mass $\sim 1.2 \mathrm{M}_{\odot}$ ). We note that rotation-induced mixing on the main sequence slightly lowers the post-dredge-up ${ }^{12} \mathrm{C} /{ }^{13} \mathrm{C}$ value, while thermohaline mixing leads to a further decrease of the carbon isotopic ratio at the luminosity of the bump, i.e. at $\log \left(L / L_{\odot}\right) \sim 2$. We see that the theoretical and observational behaviours are in complete agreement all along the evolutionary sequence. While the dispersion for stars that have not yet reached the RGB bump (i.e. with $\log \left(L / L_{\odot}\right)$ between $\sim 0.7$ and 1.8 ) reflects the dispersion in the initial rotation velocity, the data for more evolved stars requires the occurrence of thermohaline mixing as predicted by the models. 


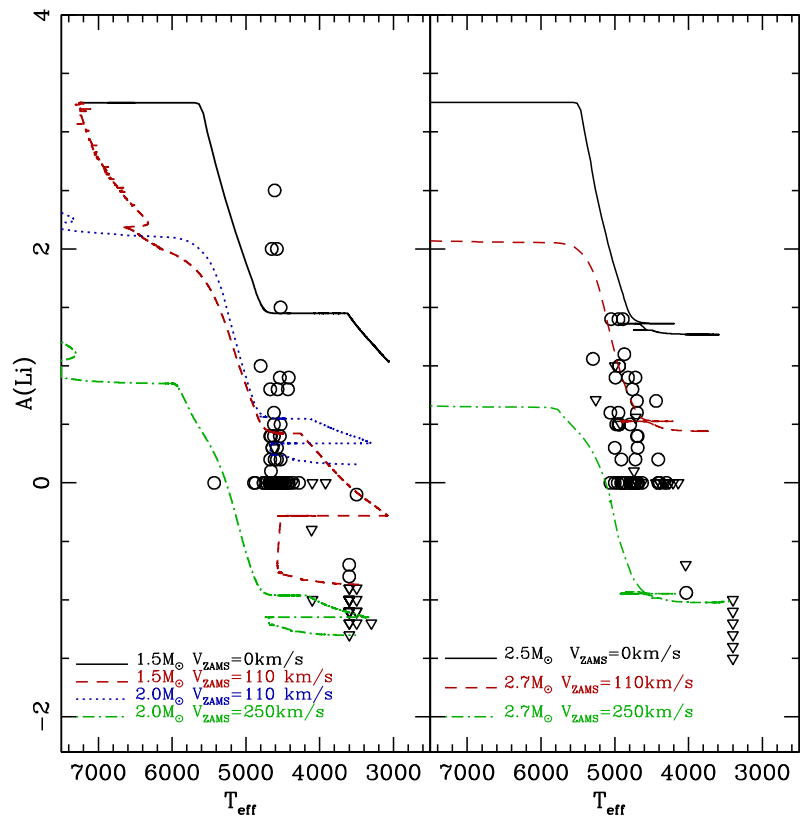

Figure 2. Lithium data for field evolved stars from the sample of Charbonnel et al. (in prep.) that are segregated according to their mass: left and right panels include respectively sample stars with masses lower and higher than $2 \mathrm{M}_{\odot}$; Li detections and upper limits are shown as circles and triangles respectively. Theoretical lithium evolution is shown from the ZAMS up to the end of the early-AGB. Various lines correspond to predictions for stellar models of different masses computed with or without rotation as indicated, and with thermohaline mixing in all cases. Figure extracted from [13].

\subsection{Lithium}

In Fig. 2 we present, as additional constraints, observed $\mathrm{Li}$ abundances that we obtained for a large sample of field red giant stars (subgiant, RGB, and early-AGB stars) with metallicities around solar, for which Hipparcos parallaxes are available and thus their evolutionary status. On the main sequence and early-RGB, rotation-induced processes lead to stronger Li depletion than in the standard case, in agreement with observations, and the observed Li dispersion reflects the dispersion in the initial rotation velocity (see also $[1,14,15])$. After the end of the first dredge-up $\left(T_{\text {eff }} \sim 4800 \mathrm{~K}\right)$, the Li abundance remains temporarily constant. For stars with mass lower than $2.0 \mathrm{M}_{\odot}$, thermohaline mixing becomes efficient at the bump in the luminosity function (which corresponds here to $T_{\text {eff }} \sim 4200 \mathrm{~K}$ ), and the $\mathrm{Li}$ abundance is then predicted to drop again in a drastic manner, explaining very well the Li upper limits obtained for the brightest RGB and AGB sample stars.

For more massive stars (right panel of Fig. 2), Li observations are compared to predictions for the 2.5 and $2.7 \mathrm{M}_{\odot}$ models. In these objects, no thermohaline mixing occurs on the too short RGB phase, and rotation-induced mixing alone explains very well the data.

\section{GLOBAL ASTEROSEISMIC QUANTITIES}

Figure 3 presents the evolutionary tracks in the Hertzsprung-Russel diagram for all models computed by [9], with and without rotation, and including effects of thermohaline instability. For the whole grid in mass and metallicity, we provide the value of different global asteroseismic quantities: large frequency separation $(\Delta v)$, frequency at maximum power $\left(v_{\max }\right)$, amplitude at maximum $\left(A_{\max }\right)$, the acoustic radii at the base of the convective envelope, and at the location 
EPJ Web of Conferences

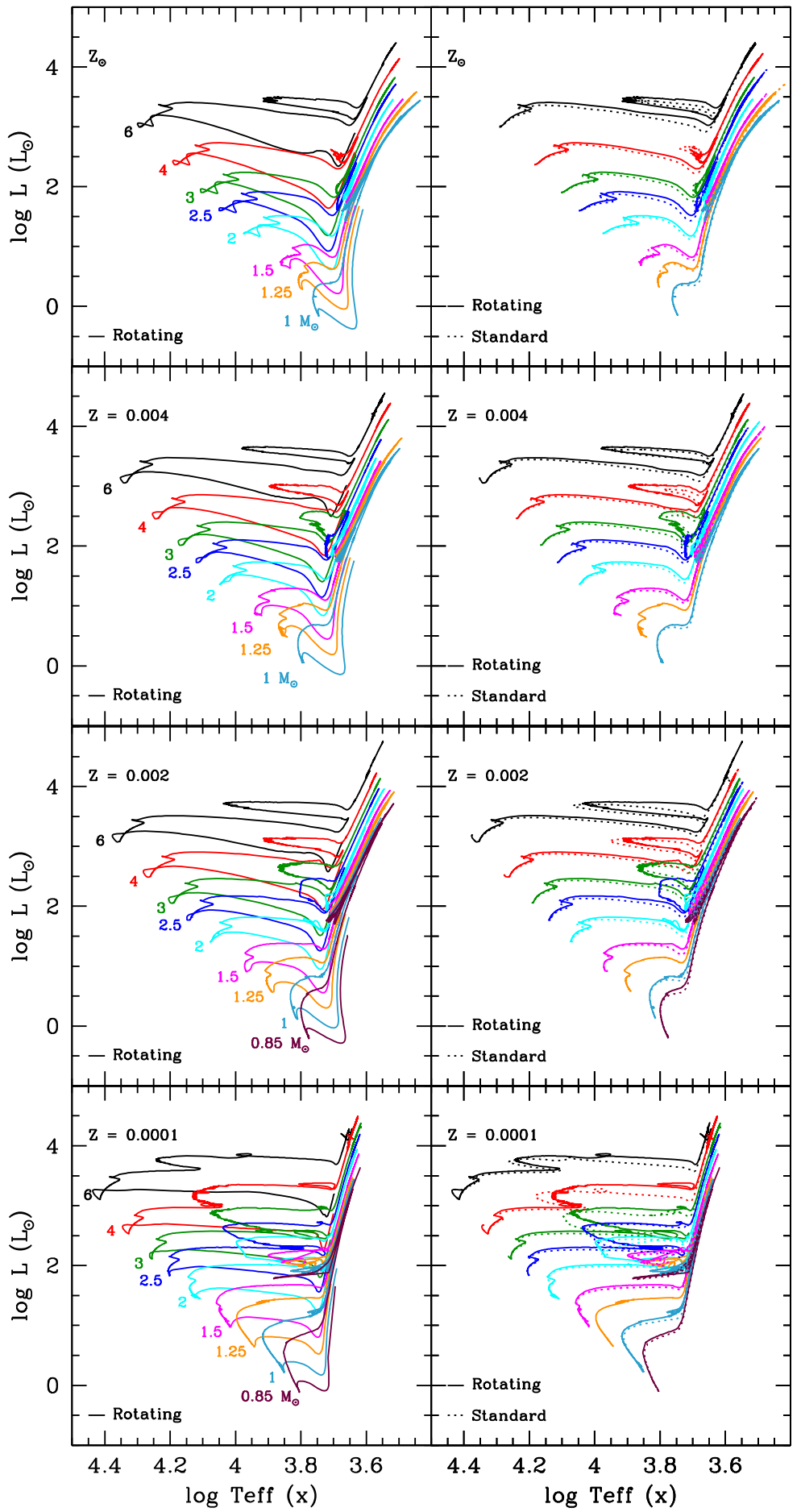

Figure 3. Theoretical evolution tracks in the HR diagram for all the calculated models for different metallicities $\left(Z_{\odot}, \mathrm{Z}=0.004, \mathrm{Z}=0.002\right.$, and $\mathrm{Z}=0.0001$, from top to bottom). In the left panels, the tracks are shown for the rotating models from the pre-main sequence on. In the right panels, both standard and rotating predictions are shown (dashed and solid lines respectively) from the ZAMS and beyond. Figure from [6]. 

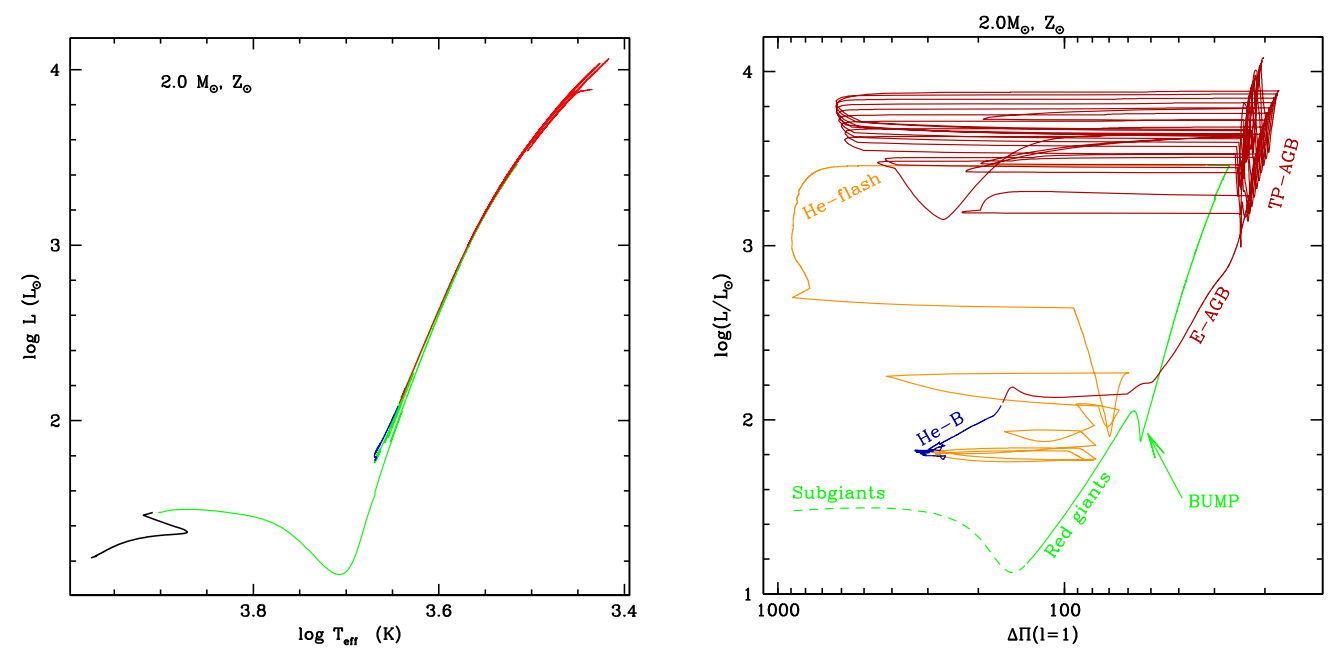

Figure 4. Left panel: the stellar evolution of a $2.0 \mathrm{M}_{\odot}$ model with solar metallicity in the HR diagram. Right panel: the stellar luminosity as a function of the asymptotic period spacing of g-modes for the standard $2.0 \mathrm{M}_{\odot}$ model with solar metallicity. Evolutionary phases are color-labeled as follows: subgiant in green dashed lines, red giant in green solid lines, helium-flash episode in orange, helium-burning in blue, and asymptotic giant branch in red. Figure from [6].

of the helium second-ionisation zone, the total acoustic radius, and the asymptotic period spacing of g-modes. These quantities have been computed with the scaling relations $\left(\Delta v, v_{\max }\right.$, and $\mathrm{A}_{\max }$ ), and with asymptotic relations (acoustic radii, $\Delta \Pi(1=1)$, and $\Delta v$ ), and are available on the following websites: http://cdsarc.u-strasbg.fr/viz-bin/qcat?J/A+A/543/A108 and http://obswww . unige.ch/Recherche/evol/-Database.

\subsection{The asymptotic period spacing of g-modes}

Figure 4 shows the asymptotic period spacing of gravity modes $\Delta \Pi(1=1)$ for the standard model of $2 \mathrm{M}_{\odot}$ at solar metallicity, without rotation. As stated by [11] and [12], this asteroseismic quantity allows to determine the evolutionary status of stars. Indeed, at $\log \left(L / L_{\odot}\right) \sim 2$ on the HR diagram, RGB and clump stars have very similar effective temperatures, while on this asteroseismic diagram $\Delta \Pi(\ell=1)$ is equal to $55 \mathrm{~s}$ at the RGB bump and $\Delta \Pi(\ell=1)=190 \mathrm{~s}$ in the clump.

\subsection{Effects of rotation-induced mixing and thermohaline mixing on global asteroseismic quantities}

As thermohaline mixing solely affects the abundances at both the surface and the external layers of hydrogen-burning shell pattern during the giant phase, without modifying the temperature, radius, luminosity, or mass of the models, this process has no impact on the global asteroseismic parameters. However, it leads to additional mixing allowing an agreement with spectroscopic observations of surface abundances (see §2).

On the other hand, rotation modifies the position of evolutionary tracks in the HR diagram (see Eggenberger et al. in this volume). Consequently, there are differences in both the maximal amplitude and the large separation between standard and rotating models (see Fig. 5). This effect clearly shows up at the end of the main sequence and during the subgiant branch. 

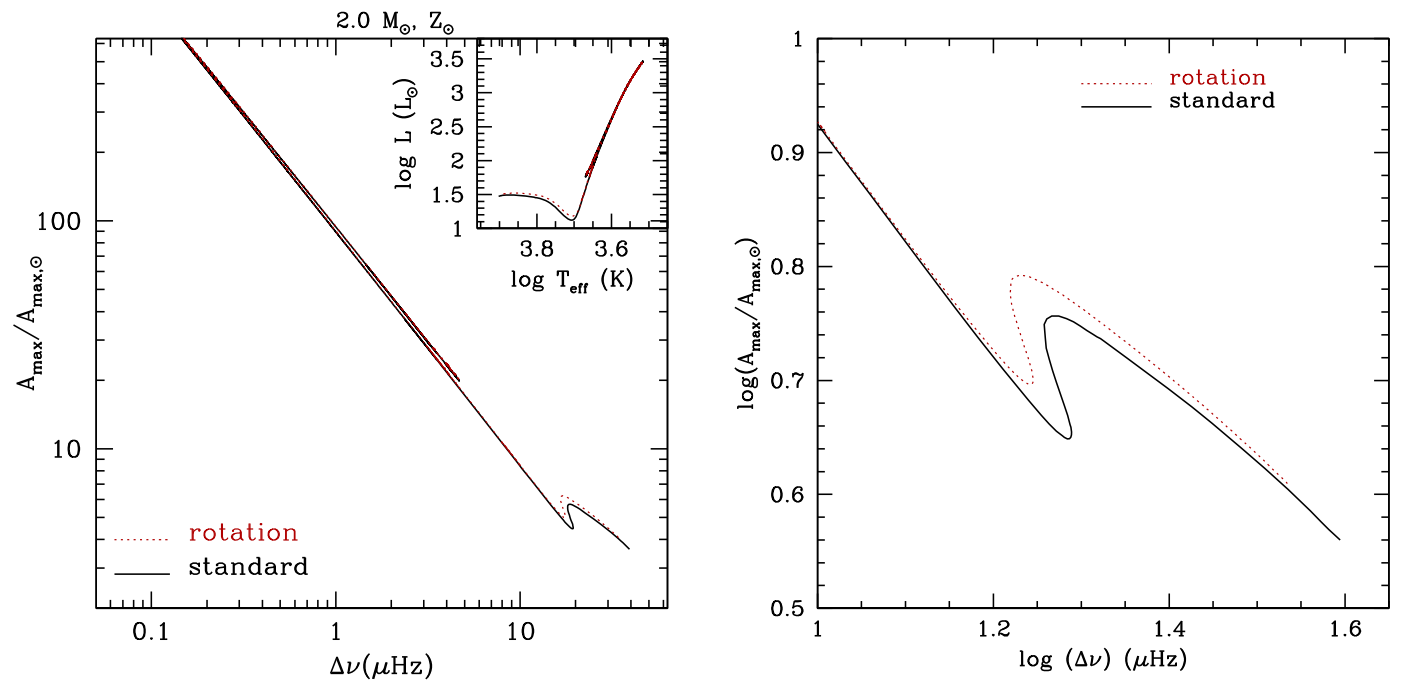

Figure 5. Maximal amplitude compared to solar one as a function of the large frequency separation for a $2.0 \mathrm{M}_{\odot}$ model with solar composition, with and without rotation, respectively in red dotted line and in black solid line: the left panel shows the stellar evolution along the red giant branch, and the right panel shows the stellar evolution around the HR gap.

\section{CONCLUSIONS}

Thermohaline instability does not change stellar parameters such as luminosity and effective temperature, nor the asteroseismic properties presented here (e.g. [6]). However, it changes significantly the surface abundances of RGB stars brighter than the bump luminosity and explains the chemical properties of this kind of giant stars. On the other hand, asteroseismic quantities which allows the determination of the evolutionary phase of stars are affected by rotation.

New spectroscopic observations of field stars for which asteroseismic observations are available (see Morel et al. in this volume) will provide a very good way to constrain stellar evolution models.

\section{References}

[1] Palacios, A., Talon, S., Charbonnel, C., Forestini, M., A\&A, 399, (2003) 603

[2] Palacios, A., Charbonnel, C., Talon, S., Siess, L., A\&A, 453, (2006) 261

[3] Charbonnel, C., Zahn, J.-P., A\&A, 467, (2007b) L15

[4] Eggleton, P.P., Dearborn, D.S.P., Lattanzio, J.C., Science, 314, (2006) 1580

[5] Lagarde, N., Charbonnel, C., Decressin, T., Hagelberg, J., A\&A, 536, (2011) A28

[6] Lagarde, N., Romano, D., Charbonnel, C., Tosi, M., Chiappini, C., Matteucci, F., A\&A, 542, (2012) A62

[7] Baglin A., Auvergne M., Boisnard L., et al., 36th COSPAR Scientific Assembly, 36, (2006) 3749

[8] Borucki W.J., Koch D., Basri G., et al., Science, 327, (2010) 977

[9] Lagarde, N., Decressin, T., Charbonnel, C., Eggenberger, P., Ekström, S., Palacios, A., A\&A, 543, (2012) A108

[10] Gilroy, K.K., Brown, J.A., ApJ, 371, (1991) 578

[11] Bedding, T.R., Mosser, B., Huber, D., et al., Nature, 471, (2011) 608

[12] Mosser, B., Barban, C., Montalbán, J., et al., A\&A, 532, (2011) A86

[13] Charbonnel, C., Lagarde, N., A\&A, 522, (2010) A10

[14] Charbonnel, C., Talon, S., A\&A, 351, (1999) 635

[15] Smiljanic, R., Pasquini, L., Charbonnel, C., Lagarde, N., A\&A, 510, (2010) A50+ 